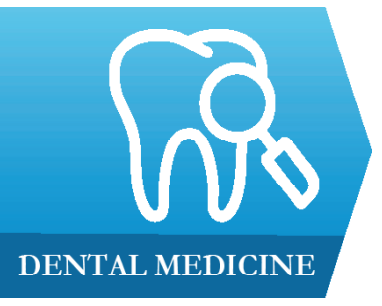

1) Department of Orthodontics and Dentofacial Orthopedics, Iuliu Hatieganu University of Medicine and Pharmacy, Cluj-Napoca, Romania

2) Department of Oral Rehabilitation, Maxillofacial Surgery and Implantology, Iuliu Hatieganu University of Medicine and Pharmacy, Cluj-Napoca, Romania

3) Department of Pharmaceutical Physics-Biophysics, Iuliu Hatieganu University of Medicine and Pharmacy, Cluj-Napoca, Romania

4) Department of Prosthetic Dentistry and Dental Materials, Iuliu Hatieganu University of Medicine and Pharmacy, Cluj-Napoca, Romania

5) Department of Radiology, Iuliu Hatieganu University of Medicine and Pharmacy, Cluj-Napoca, Romania

6) Department of Oral Radiology, Iuliu Hatieganu University of Medicine and Pharmacy, Cluj-Napoca, Romania

7) Department of Cranio-Maxillofacial Surgery, Iuliu Hatieganu University of Medicine and Pharmacy, Cluj-Napoca, Romania

DOI: $10.15386 / \mathrm{mpr}-1434$

Manuscript received: 29.07.2019

Accepted: 21.08.2019

Address for correspondence:

mhedesiu@gmail.com

\title{
Clinical indications and radiation doses of cone beam computed tomography in orthodontics
}

Ioana Maria Colceriu-Şimon ${ }^{1}$, Mihaela Băciuț², Rareş Ionuţ Ştiufiuc ${ }^{3}$, Alexandra Aghiorghiesei ${ }^{4}$, Viorica Țărmure ${ }^{1}$, Manuela Lenghel ${ }^{5}$, Mihaela Hedeşiư ${ }^{6}$, Grigore Băciuţ ${ }^{7}$

\begin{abstract}
Cone beam computed tomography can be used in pediatric population when a tridimensional analysis of dental and maxillofacial bone structures is required. Even though $\mathrm{CBCT}$ is considered a low dose radiological examination, ionizing radiation is a known human carcinogenic factor. Furthermore, biological effects are more important in young patients because of their higher radiosensitivity. Orthodontic treatment is typically initiated at young ages and the most common radiographs at this age are dental. This makes it important to quantify the effects of diagnostic radiographs, in particular of CBCT, due to the fact that the radiation doses are higher compared to conventional radiological methods. So far, the carcinogenic response after low dose radiation exposure is not fully understood in the scientific literature. The aim of our review was to emphasize the main indications of CBCT in orthodontics and to evaluate the radiation doses and potential risks of CBCT irradiation of pediatric patients.
\end{abstract}

Keywords: cone beam computed tomography, ionizing radiation, dosimetry, orthodontics, pediatric population

\section{Introduction}

Cone beam computed tomography (CBCT) can be used in pediatric population when a tridimensional (3D) representation of dental and maxillofacial bone structures is required [1]. CBCT has several advantages compared to twodimensional (2D) radiographs for the diagnosis and treatment planning in the field of orthodontics and dento-facial orthopedics [2], but the resulting radiation doses are also higher. The use of CBCT is increasing more and more, with some orthodontists completely replacing 2D radiographic images with 3D ones. For this reason, the clinical indications for this investigation, but also the irradiation doses are becoming intensely debated in the scientific literature. Stochastic effects, such as genetic mutations and carcinogenesis, resulting from low doses of radiation may occur many years after irradiation if the harmful effects are not properly repaired by the human body. Furthermore, young patients are more susceptible to ionizing radiation (IR) because of the increased number of young cells that are strongly affected at the level of the DNA and cell division. This problem raises the need to investigate the effects of low dose IR, such as CBCT exposure, especially because of the increased proportion of children in the orthodontic practice. The evaluation of the effects of irradiation remains an important issue to solve in order to understand the benefits and risks of radiological exposure in the orthodontic field.

The purpose of our review was to summarize evidence-based guidelines regarding the acquisition of $\mathrm{CBCT}$ scans for orthodontic treatment; to evaluate the CBCT radiation doses in orthodontics and to discuss the potential harmful effects of $\mathrm{CBCT}$ radiation.

\section{Indications for CBCT use in orthodontics}

The objective of orthodontics and dento-facial orthopedics is the treatment of dental and skeletal disharmony in order to improve aesthetic aspects and function [1]. The initial orthodontic documentation includes typically 2D images, such as intraoral, panoramic or 
lateral cephalometric radiograps [2]. CBCT was introduced in various branches of dentistry for clinical situations requiring the representation of anatomical structures in the third dimension [3]. CBCT has several advantages over 2D imaging, such as an increased image quality, a short exposure time, and a lower radiation dose compared to traditional computed tomography [4]. However, although the use of CBCT is increasing steadily, the effects of additional irradiation associated with its frequent indication are also becoming a controversial topic.

The routine use of CBCT for all orthodontic patients is currently not supported by strong evidence, leading to an intense debate in the scientific literature. Some practitioners advocate its routine use for all orthodontic patients, while others are more reluctant because of the increased radiation burden to the pediatric patients. For example, a debate on this subject was published in 2012 by the American Journal of Orthodontics and Dentofacial Orthopedics (AJO-DO) [5,6].

Multiple international organizations have tried to review the available literature and to offer guidelines for a judicious use of CBCT in this field. The American Academy of Oral and Maxillofacial Radiology (AAOMR) and the British Orthodontic Society recommend it only for specific clinical cases where 2D radiological imaging cannot provide sufficient diagnostic information, such as cleft palate cases, impacted or supernumerary teeth and orthognathic surgery planning [1,7]. In other situations, such as evaluation of the alveolar bone quantity, airway assessment, temporomandibular joint pathology and for placement of temporary anchorage devices, the benefits of CBCT examination have to be compared to the risks of IR [1].

The European evidence-based guidelines based on a systematic review and published in 2012, known as SedentexCT Project, have concluded that there is a lack of evidence of significant clinical impact regarding the use of large volume CBCT for the routine orthodontic diagnosis and treatment planning [8]. Another group of specialists in the field of radiology and orthodontics, the European DIMITRA project (dentomaxillofacial paediatric imaging: an investigation towards low-dose radiation induced risks), is focused on optimizing pediatric doses. In its report from 2018, the main CBCT indications in pediatric dentistry, such as impacted/supernumerary teeth, dentoalveolar trauma, orofacial clefts, dental anomalies, surgical planning of autotransplantation and syndromes have been provided [9].

One more recent report about clinical considerations and potential liability associated with the use of IR in orthodontics has been published in AJO-DO, aiming to discuss guidelines for radiographic acquisition at different points along the orthodontic treatment timeline. The conclusion of this article was to prescribe necessary radiographs only after clinical examination and only if justified and to consider CBCT imaging when it is expected to offer a benefit or change to the treatment result of the patient as compared to $2 \mathrm{D}$ radiographs [10].

\section{Radiation doses of 2D and 3D diagnostic imaging in orthodontics}

In order to compare radiation doses of different diagnostic imaging techniques and to estimate the risk of radiation to the human body, the effective dose of radiation exposure is utilized and measured in milisieverts $(\mathrm{mSv}) /$ microsieverts $(\mu \mathrm{Sv})$. The effective dose is calculated by multiplying the absorbed organ dose with a weighting factor for a specific tissue or organ. The tissue/organ weighting factor depends on several factors, such as the type and sensitivity of the irradiated tissue and is provided and updated by the International Commission on Radiological Protection (ICPR) [11]. For the calculation of the effective dose for imaging of the head, the dose to the thyroid contributes the most due to its increased radiosensitivity. In 2007, an update of the ICPR report considered also the salivary glands separately due to their location in the field of view of the head and neck region. By receiving their own weighting factor, the salivary glands contributed to a considerable increase of the effective dose [12].

Some studies on the role of medical radiological diagnosis in head and neck cancers do not take into account the dental sources of diagnostic irradiation. The average annual effective dose from natural sources for all humans on Earth is estimated to be $2400 \mu \mathrm{Sv}$. A panoramic radiograph may be associated with an effective dose the same as 1 to 5 days additional background radiation, while the dental CBCT risk could result in an effective dose equivalent to a few days up to a couple of months of background radiation, depending on the type of the machine and clinical protocol used [13].

$\mathrm{CBCT}$ radiation doses presented in the literature vary widely, depending on the type of the used CBCT machine and the exposure factors. Among them, imaging parameters (kilovoltage $(\mathrm{kV})$, milliampere seconds (mAs)); voxel size (spatial resolution); field of view (FOV; limited or full); and exposure time influence the radiation dose [14].

A larger FOV or a higher spatial resolution when other exposure parameters ( $\mathrm{kV}$ and $\mathrm{mAs})$ are kept the same lead to a higher patient radiation dose [15]. The mandibular FOV has a larger dose than the maxillary [16], because the salivary glands, thyroid, and esophagus are more irradiated in this examination approach. Selection of different technical factors for a higher or lower image quality can result in up to seven fold differences in dose $[17,18]$. The challenge posed by $\mathrm{CBCT}$ is to reduce radiation doses but without decreasing the image quality or diagnostic information. Therefore, this can be done either by reducing the FOV or by reducing exposure parameters for clinical situations where there is no need for a very detailed image quality, such as for checking the angulation of the roots [19].

When radiographing children, it is important to take into account the radiation protection principles: justification, optimization and dose limitation. Regarding optimization and dose limitation, it is important to know the technical aspects of the CBCT machines that are used. Some of them 
have exposure parameters, such as $\mathrm{kV}, \mathrm{mA}$, and exposure time established by the manufacturer and they cannot be adjusted for every patient [20]. If the irradiation parameters are not reduced for a pediatric exposure, the radiation doses for children may exceed typical adult radiation levels due to differences in organ sizes and susceptibility to radiation. If CBCT is utilized, the smallest possible FOV that shows the region of interest should be chosen for children in order to reduce the radiation doses [21].

Published dosimetry data are available only for some of the CBCT machines available on the market. There is a need of studies regarding irradiation dose optimization that consider patient factors, image quality and acceptable dose levels in controlled settings. The majority of studies for radiation dose calculation and radiation risk estimation are based on thermoluminescent dosimetry (TLD) techniques using anthropomorphic phantoms and present a variety of methodology, especially regarding the type of phantom used (child, adolescent or adult) and TLD number and positioning. Measurements on child and adult anthropomorphic phantoms give different results because of differences in the size of the subjects, the location of organs and the exposure protocol. For instance, the thyroid in a child is exposed directly during CBCT irradiation because the distance between the thyroid and the lower border of the mandible in a child is reduced. Furthermore, it has been shown that effective doses for the salivary glands are approximately $30 \%$ higher for young children than for adolescents [22]. Different exposure protocols showed that child phantom effective doses were approximately $36 \%$ greater than adult phantom doses [19]. Due to the increased radiosensitivity of children, the risks of harmful health effects are an additional 2 to 5 times higher compared to adults [23].

A software alternative for calculating organ doses are Monte Carlo (MC) dose simulation studies using computational phantoms. Efforts are focused on the calculation of radiation doses depending on the age of the patients, especially for the pediatric population, which cannot be represented by only one average phantom like adults, because their organs change in size and shape with age. Andreas et al. have developed adatabase of pediatric head voxel models that covers the age range from 2 months to 14 years in order to calculate doses using MC simulations. Their simulation study showed absorbed organ dose differences larger than 50\% among the 5, 8 and 12 years old models when exposed to the same parameters [24]. Oenning et al. described the development of agespecific phantoms for age specific $\mathrm{CBCT}$ image quality assessment and optimization [25].

The range of effective doses of small, medium and large FOV using the ICPR 2007 recommendations are presented in Table I. A small FOV is defined by an area $\leq 10 \mathrm{~cm}$ that captures most of one or both arches, but not all of the anatomy of the maxilla; a medium FOV by an area of $10-15 \mathrm{~cm}$ that captures the entire dentition and temporomandibular joints, while a large FOV by an area $>15 \mathrm{~cm}$ that captures the maxillofacial complex, chin and nose. The studies have showed that the dose ranges have a considerable variation from one $\mathrm{CBCT}$ examination and from one $\mathrm{CBCT}$ machine to another.

A direct comparison between the effective doses of 2D and 3D radiological examinations have been studied [28-30]. The results showed an effective dose for panoramic radiography of about $22.0 \mu \mathrm{Sv}$, for a lateral cephalometric examination about $4.5 \mu \mathrm{Sv}$ and for a CBCT examination 61 to $134 \mu \mathrm{Sv}$. Another study have showed that a typical panoramic and cephalometric orthodontic examination will expose the patient to 7.5 to $25.4 \mu \mathrm{Sv}$ effective dose (with salivary glands) [14]. Other studies that analyzed just the effective dose of conventional dental radiography estimated the dose for a panoramic radiograph ranging from 3.85 to $38.0 \mu \mathrm{Sv}$ and for a lateral cephalometric examination from 1.1 to $5.6 \mu \mathrm{Sv}[31,32]$.

Table I. Effective dose ranges for small, medium and large FOVs, for adult and children anthropomorphic phantoms.

\begin{tabular}{|c|c|c|}
\hline FOV & $\begin{array}{c}\text { Effective dose } \\
\text { for adult patients }(\mu \mathrm{Sv})\end{array}$ & $\begin{array}{c}\text { Effective dose } \\
\text { for children ( } \mu \mathrm{Sv})\end{array}$ \\
\hline Large & $\begin{array}{c}68-1073[18] \\
68-368 \text { (average 131) [26] } \\
46-1073(\text { average } 212)[27] \\
68-1073[4]\end{array}$ & \multirow{2}{*}{$\begin{array}{c}\text { 13-769 (average 175) [27] } \\
\text { 114-282 (10 years old model) [22] } \\
\text { 81-216 (adolescent model) [22] }\end{array}$} \\
\hline Medium & $\begin{array}{c}69-560[18] \\
\text { 28-265 (average 88) [26] } \\
9-560 \text { (average 177) [27] } \\
\quad 48-560[4]\end{array}$ & \\
\hline Small & $\begin{array}{l}189-652[18] \\
\text { 19-44 (average 34) [26] } \\
\text { 5-652 (average 84) [27] } \\
\text { 28-674 [4] }\end{array}$ & $\begin{array}{c}5-582 \text { (average 103) [27] } \\
<35[22]\end{array}$ \\
\hline
\end{tabular}


Even though $\mathrm{CBCT}$ is considered a low dose radiological method [27], the effective dose of CBCT is several to hundreds of times higher compared with conventional dental radiography [33]. A study by Signorelli et al. showed that although one CBCT scan may replace all conventional orthodontic records, the irradiation is still 2 to 4 times higher with CBCT. Depending on the scanning parameters, the radiation dose of a CBCT is about 3 to 6 times a digital panoramic radiograph and 15 to 26 times a lateral cephalogram [34]. Ludlow et al. stated that the irradiation dose with a NewTom $3 \mathrm{G} \mathrm{CBCT}$ machine is 2 to 4.5 higher than doses of a conventional ortopantomography or lateral cephalometry. In sum, it was estimated that the radiation doses for a large FOV CBCT scan are 3 to 7 times greater than the radiation doses from panoramic radiographs [35]. Table II presents CBCT doses with different FOVs as multiples of dental panoramic examinations with an effective dose of $24.5 \mu \mathrm{Sv}$, days of per capita background dose based on an annual full body exposure of $3 \mu \mathrm{Sv}$, and probability of fatal cancer with the ICRP 2007 tissue weighting factors [32].

Table II. Detrimental effects of CBCT doses related to different field of views (FOVs).

\begin{tabular}{|l|c|c|c|} 
FOVs & $\begin{array}{c}\text { Dose as multiple } \\
\text { of typical } \\
\text { panoramic dose }\end{array}$ & $\begin{array}{c}\text { Days of per capita } \\
\text { background } \\
\text { radiation, } \\
\text { ICRP 2007 }\end{array}$ & $\begin{array}{c}\text { Probability of } \\
\mathbf{x} \text { in a million } \\
\text { fatal cancer }\end{array}$ \\
\hline Large & $3-44$ & $8-131$ & $4-59$ \\
Medium & $4-35$ & $9-105$ & $4-47$ \\
Small & $8-27$ & $23-79$ & $10-36$
\end{tabular}

\section{Risks of low dose CBCT irradiation}

IR can induce harmful biological effects, depending on radiation dose, age at exposure, the radiosensitivity of the organ or genetic factors [36]. IR produces single or double strand breaks in the DNA helix, the latter being the principal lesion that can lead to cell death. Another mechanism of damage are the residual double strand breaks after inefficient repair mechanisms that may result in mutations or genomic disturbances in a cell that survives. Over time, these could lead to genomic instability and result in malignant cell transformation [37].

Studies of individuals exposed to IR showed two potential types of radiation damage. Deterministic effects occur after high doses of radiation and only after a threshold has been reached. After that, the effects are proportional to the dose of radiation. On the other hand stochastic effects can be a consequence of lower levels of radiation. These occur without a threshold for radiation damage, but the probability of an effect increases proportional to the radiation dose. Thus, there is a probability that an unrepaired damage to the cell nucleus will lead to carcinogenesis at later times during life after exposure [38].
Taking the effective dose of dental sources of diagnostic irradiation into consideration is necessary because young patients have a higher risk of developing cancer than adults. The young age at which orthodontic treatment is typically initiated and the fact that the most common radiographs at this age are dental makes it important to quantify the effect of diagnostic radiographs [2]. The pediatric population, consisting of patients under 18 years, shows increased radiosensitivity compared to adults due to the higher rates of cell growth and organ development. Children's susceptibility to mutagenic factors is increased due to differences in assimilation, metabolism and excretion. Also, children have a longer lifespan to express the radiation induced effects [39].

In the last years, several studies evaluated the risk related to low dose radiation exposure, the most investigated being the epidemiological Life Span Study of Japanese atomic bomb survivors [40]. The results indicated that the greatest risk of thyroid cancer was found among those exposed before the age of 10 years, and the highest risk was seen 15 to 29 years after exposure and was still increased 40 years after exposure [41]. A study published in 2010 concluded that exposure to dental X-rays, particularly multiple exposures, may be associated with an increased risk of thyroid cancer [42].

For a long time, the linear no-threshold model (LNT) model was adopted for the estimation of the effects of low doses of radiation. According to this model applied for stochastic effects, all doses regardless of how low they are must be considered potential carcinogenic [38]. The standards used for protection against low levels of IR were decided by extrapolating from cancer risks observed following exposure to intermediate or high doses. The LNT model used in radioprotection for the quantification of the radiation exposure at high doses was considered by many authors not being suitable for the estimation of the effects at low doses of radiation [43] and the validity of this model is intensely debated in the literature. However, there is not yet another suitable model that analyzes the effects of low doses of IR.

The CBCT irradiation dose is greater than doses from a panoramic or cephalometric radiological examination, even with the lowest radiation dose CBCT machine used. The excess risk of cancer is tried to be estimated by using the effective dose, but most of the calculations are made on adults. The risk of carcinogenesis or development of other stochastic effects using the ICRP probability coefficient of $6.0 \times 10^{-2} \mathrm{~Sv}^{-1}$ are: 0.3 to $1.3 \times 10^{-6}$ for a dental panoramic radiograph, $0.1-0.2 \times 10^{-6}$ for cephalometric radiography and 3.5 to $61.5 \times 10^{-6}$ for full FOV CBCT exposure $[11,14]$. It is estimated that children may be at least 3 times more sensitive to radioinduced malignancies compared to adults, but there are limited studies examining the radiation dose/exposure in dentomaxillofacial area of pediatric patients [44]. 
There is no scientifically proven risk of cancer development following $\mathrm{CBCT}$ exposure in the pediatric population, due to the fact that most of the studies on radiation related risks are performed on an adult population. Therefore, because stochastic effects increase with the irradiation dose and are expressed over many years, the "as low as reasonably achievable" (ALARA) principle is recommended also for the use of CBCT in orthodontics [33].

\section{Conclusion}

Ionizing radiation is a known human carcinogenic factor and its biological effects are more important in young patients because of their higher radiosensitivity. The effects of ionizing radiation in pediatric patients after CBCT exposures and the cumulative risk of radiation needs to be further studied and all the indications for CBCT in orthodontic procedures should be very well justified by strong scientific evidence. By evaluating the advantages and risks of CBCT and based on specialized and updated literature, this article aimed to discuss CBCT use in orthodontics and to emphasize the responsibility of the orthodontist towards a discerning use of $\mathrm{CBCT}$ in daily practice.

\section{Acknowledgements}

This research was funded by Iuliu Hațieganu University of Medicine and Pharmacy, Cluj-Napoca, Romania, under Grant Project PCD "Study of the biological effects of Oral diagnostic irradiation with Raman Spectroscopy", number 7690/106/15/06/2016.

\section{References}

1. American Academy of Oral and Maxillofacial Radiology. Clinical recommendations regarding use of cone beam computed tomography in orthodontics. [corrected]. Position statement by the American Academy of Oral and Maxillofacial Radiology. Oral Surg Oral Med Oral Pathol Oral Radiol. 2013;116:238-257.

2. Hujoel P, Hollender L, Bollen AM, Young JD, McGee M, Grosso A. Radiographs associated with one episode of orthodontic therapy. J Dent Educ. 2006;70:1061-1065.

3. Kapila S, Conley RS, Harrell WE Jr. The current status of cone beam computed tomography imaging in orthodontics. Dentomaxillofac Radiol. 2011;40:24-34.

4. Lorenzoni DC, Bolognese AM, Garib DG, Guedes FR, Sant'Anna EF. Cone-beam computed tomography and radiographs in dentistry: aspects related to radiation dose. Int J Dent. 2012;2012:813768.

5. Halazonetis DJ. Cone-beam computed tomography is not the imaging technique of choice for comprehensive orthodontic assessment. Am J Orthod Dentofacial Orthop. 2012;141:403,

6. Larson BE. Cone-beam computed tomography is the imaging technique of choice for comprehensive orthodontic assessment. Am J Orthod Dentofacial Orthop. 2012;141:402, 404, 406, 408, 410

7. Turpin DL. British Orthodontic Society revises guidelines for clinical radiography. Am J Orthod Dentofacial Orthop.
2008;134:597-598.

8. The SEDENTEXCT project. Radiation protection: cone beam CT for dental and maxillofacial radiology. Evidence based guidelines. Geneva, Switzerland: European Commission; 2011. Available from: http://www.sedentexct. eu/files/radiation_protection_172.pdf

9. Oenning AC, Jacobs R, Pauwels R, Stratis A, Hedesiu M, Salmon B, et al. Cone-beam CT in paediatric dentistry: DIMITRA project position statement. Pediatr Radiol. 2018;48:308-316.

10. Abdelkarim A, Jerrold L. Clinical considerations and potential liability associated with the use of ionizing radiation in orthodontics. Am J Orthod Dentofacial Orthop. 2018;154:15-25.

11. 1990 recommendations of the International Commission on Radiological Protection. Ann ICRP. 1991;21:1-201.

12. The 2007 Recommendations of the International Commission on Radiological Protection. ICRP publication 103. Ann ICRP. 2007;37:1-332.

13. United Nations Scientific Committee on the Effects of Atomic Radiation. Sources and effects of ionizing radiation. UNSCEAR 1993 Report to the General Assembly with scientific annexes. New York: United Nations; 1993. Available from: https://www.unscear.org/docs/ publications/1993/UNSCEAR_1993_Report.pdf

14. Brooks SL. CBCT Dosimetry: Orthodontic Considerations. Semin Orthod 2009;15(1):14-18.

15. Li G. Patient radiation dose and protection from cone-beam computed tomography. Imaging Sci Dent. 2013;43:63-69.

16. Ludlow JB, Davies-Ludlow LE, Brooks SL. Dosimetry of two extraoral direct digital imaging devices: NewTom cone beam CT and Orthophos Plus DS panoramic unit. Dentomaxillofac Radiol. 2003;32:229-234.

17. Watanabe H, Honda E, Tetsumura A, Kurabayashi T. A comparative study for spatial resolution and subjective image characteristics of a multi-slice CT and a cone-beam CT for dental use. Eur J Radiol. 2011;77:397-402.

18. Ludlow JB, Ivanovic M. Comparative dosimetry of dental CBCT devices and 64-slice CT for oral and maxillofacial radiology. Oral Surg Oral Med Oral Pathol Oral Radiol Endod. 2008;106:106-114.

19. Ludlow JB, Walker C. Assessment of phantom dosimetry and image quality of i-CAT FLX cone-beam computed tomography. Am J Orthod Dentofacial Orthop. 2013;144:802-817.

20. Pauwels R, Araki K, Siewerdsen JH, Thongvigitmanee SS. Technical aspects of dental CBCT: state of the art. Dentomaxillofac Radiol. 2015;44:20140224. doi: 10.1259/ dmfr.20140224.

21. Palomo JM, Rao PS, Hans MG. Influence of CBCT exposure conditions on radiation dose. Oral Surg Oral Med Oral Pathol Oral Radiol Endod. 2008;105:773-782.

22. Theodorakou C, Walker A, Horner K, Pauwels R, Bogaerts R, Jacobs R, et al. Estimation of paediatric organ and effective doses from dental cone beam CT using anthropomorphic phantoms. Br J Radiol. 2012;85:153-160.

23. Brenner DJ, Elliston C, Hall E, Berdon W. Estimated Risks of Radiation-Induced Fatal Cancer from Pediatric CT. AJR 
Am J Roentgenol. 2001;176:289-96.

24. Stratis A, Touyz N, Zhang G, Jacobs R, Bogaerts R, Bosmans H, et al. Development of a paediatric head voxel model database for dosimetric applications. Br J Radiol. 2017;90:20170051.

25. Oenning AC, Salmon B, Vasconcelos KF, Pinheiro Nicolielo LF, Lambrichts I, Sanderink G, et al. DIMITRA paediatric skull phantoms: development of age-specific paediatric models for dentomaxillofacial radiology research. Dentomaxillofac Radiol. 2018;47:20170285.

26. Pauwels R, Beinsberger J, Collaert B, Theodorakou C, Rogers J, Walker A, et al. Effective dose range for dental cone beam computed tomography scanners. Eur J Radiol. 2012;81:267-271.

27. Ludlow JB, Timothy R, Walker C, Hunter R, Benavides E, Samuelson D, et al. Effective dose of dental CBCT-a meta analysis of published data and additional data for nine CBCT units. Dentomaxillofac Radiol. 2015;44:20140197.

28. Qu XM, Li G, Ludlow JB, Zhang ZY, Ma XC. Effective radiation dose of ProMax 3D cone-beam computerized tomography scanner with different dental protocols. Oral Surg Oral Med Oral Pathol Oral Radiol Endod. 2010;110:770-776.

29. Grünheid T, Kolbeck Schieck JR, Pliska BT, Ahmad M, Larson BE. Dosimetry of a cone-beam computed tomography machine compared with a digital x-ray machine in orthodontic imaging. Am J Orthod Dentofacial Orthop. 2012; 141:436-443.

30. Silva MA, Wolf U, Heinicke F, Bumann A, Visser H, Hirsch E. Cone-beam computed tomography for routine orthodontic treatment planning: a radiation dose evaluation. Am J Orthod Dentofacial Orthop. 2008;133:640.e1-e5.

31. Gijbels F, Jacobs R, Bogaerts R, Debaveye D, Verlinden S, Sanderink G. Dosimetry of digital panoramic imaging. Part I: Patient exposure. Dentomaxillofac Radiol. 2005;34:145-149.

32. Ludlow JB, Davies-Ludlow LE, White SC. Patient risk related to common dental radiographic examinations: the impact of 2007 International Commission on Radiological Protection recommendations regarding dose calculation. J Am Dent Assoc. 2008;139:1237-1243.

33. Farman AG. ALARA still applies. Oral Surg Oral Med Oral Pathol Oral Radiol Endod. 2005;100:395-397.
34. Signorelli L, Patcas R, Peltomäki T, Schätzle M. Radiation dose of cone-beam computed tomography compared to conventional radiographs in orthodontics. J Orofac Orthop. 2016;77:9-15.

35. Ludlow JB, Davies-Ludlow LE, Brooks SL, Howerton WB. Dosimetry of 3 CBCT devices for oral and maxillofacial radiology: CB Mercuray, NewTom 3G and i-CAT. Dentomaxillofac Radiol. 2006;35:219-226.

36. Allan JM. Genetic susceptibility to radiogenic cancer in humans. Health Phys. 2008;95:677-686.

37. Willers H, Dahm-Daphi J, Powell SN. Repair of radiation damage to DNA. Br J Cancer. 2004;90:1297-1301.

38. Little MP, Wakeford R, Tawn EJ, Bouffler SD, Berrington de Gonzalez A. Risks associated with low doses and low dose rates of ionizing radiation: why linearity may be (almost) the best we can do. Radiology. 2009;251:6-12.

39. Hagmar L, Bonassi S, Strömberg U, Brøgger A, Knudsen LE, Norppa $\mathrm{H}$, et al. Chromosomal aberrations in lymphocytes predict human cancer: a report from the European Study Group on Cytogenetic Biomarkers and Health (ESCH). Cancer Res. 1998;58:4117-4121.

40. Pierce DA, Preston DL. Radiation-related cancer risks at low doses among atomic bomb survivors. Radiat Res. 2000;154:178-186.

41. Thompson DE, Mabuchi K, Ron E, Soda M, Tokunaga $\mathrm{M}$, Ochikubo $\mathrm{S}$, et al. Cancer incidence in atomic bomb survivors. Part II: Solid tumours, 1958-1987. Radiat Res. 1994;137(2 Suppl):S17-S67.

42. Memon A, Godward S, Williams D, Siddique I, Al-Saleh K. Dental $\mathrm{x}$-rays and the risk of thyroid cancer: a case-control study. Acta Oncol. 2010;49:447-453.

43. Tubiana M, Feinendegen LE, Yang C, Kaminski JM. The Linear No-Threshold Relationship Is Inconsistent with Radiation Biologic and Experimental Data. Radiology. 2009;251:13-22.

44. United Nations Scientific Committee on the Effects of Atomic Radiation. UNSCEAR 2006 Report to the General Assembly with Scientific Annexes. Effects of Ionizing Radiation. Volume I Report and Annexes A and B. New York, NY: United Nations, 2008. Available from: https:/www. unscear.org/docs/publications/2006/UNSCEAR_2006_ Report_Vol.I.pdf 